\title{
Superconductivity in single crystals of LaFePO
}

\author{
J. J. Hamlin, R. E. Baumbach, D. A. Zocco, T. A. Sayles and M. B. Maple \\ Department of Physics and Institute for Pure and Applied Physical Sciences, University of \\ California, San Diego, La Jolla, CA 92093 \\ E-mail: mbmaple@ucsd.edu
}

\begin{abstract}
Single crystals of the compound LaFePO were prepared using a flux growth technique at high temperatures. Electrical resistivity measurements reveal metallic behavior and a resistive transition to the superconducting state at a critical temperature $T_{c} \sim 6.6 \mathrm{~K}$. Magnetization measurements also show the onset of superconductivity near $6 \mathrm{~K}$. In contrast, specific heat measurements manifest no discontinuity at $T_{c}$. These results lend support to the conclusion that the superconductivity is associated with oxygen vacancies that alter the carrier concentration in a small fraction of the sample, although superconductivity characterized by an unusually small gap value can not be ruled-out. Under applied magnetic fields, $T_{c}$ is suppressed anisotropically for fields perpendicular and parallel to the $a b$-plane, suggesting that the crystalline anisotropy strongly influences the superconducting state. Preliminary highpressure measurements show that $T_{c}$ passes through a maximum of nearly $14 \mathrm{~K}$ at $\sim 110 \mathrm{kbar}$, demonstrating that significantly higher $T_{c}$ values may be achieved in the phosphorus-based oxypnictides.
\end{abstract}




\section{Introduction}

There has been a flurry of research activity following the recent reports of superconductivity with high critical temperatures $T_{c}$ in the system $\mathrm{LnFeAs}\left[\mathrm{O}_{1-x} \mathrm{~F}_{x}\right]$ where $\mathrm{Ln}$ is a lanthanide [1, 2, 3, 4, 5, 6]. To date, values of $T_{c}$ as high as $55 \mathrm{~K}$ have been reported for $\mathrm{Ln}=\mathrm{Sm}$ [5]. These compounds belong to a general class of compounds with a layered crystal structure of the form LnFePnO that were first synthesized by Jeitschko and coworkers with $\mathrm{Pn}=\mathrm{P}$ [7] and As [8]. Superconductivity in this series of materials was discovered in LaFePO in 2006 by Kamihara et al. [9], for which values of $T_{c}$ that range from $3 \mathrm{~K}$ [9] to $7 \mathrm{~K}$ [10] have been reported. However, in a recent study of polycrystalline materials, it was concluded that stoichiometric $\mathrm{LaFePO}$ is metallic but non-superconducting [11] at temperatures as low as $0.35 \mathrm{~K}$. In this paper, we report the synthesis of single crystals of LaFePO. These crystals exhibit superconducting transitions at $6.6 \mathrm{~K}$ and $6.0 \mathrm{~K}$, according to electrical resistivity and magnetic susceptibility measurements, respectively. However, there is no specific heat jump at $T_{c}$, suggesting that only a small fraction of the sample is superconducting. The superconductivity appears to be a property of the single crystals, since the resistively measured upper critical field is quite anisotropic. It is possible that the superconductivity is associated with oxygen vacancies that dope a small fraction of the compound with charge carriers. We present measurements of the electrical resistivity, magnetic susceptibility, and specific heat in the normal state. Preliminary measurements of the pressure dependence of $T_{c}$ are also reported.

\section{Experimental Details}

Single crystals of $\mathrm{LaFePO}$ were grown from elements and elemental oxides with purities $>99.9 \%$ in a molten Sn:P flux. The growths took place over a 1 week period in quartz ampoules which were sealed with 75 torr Ar at room temperature. The inner surface of each quartz ampoule was coated with carbon by a typical pyrolysis method. The starting materials were $\mathrm{La}, \mathrm{Fe}_{2} \mathrm{O}_{3}, \mathrm{P}$, and $\mathrm{Sn}$, which were combined in the molar ratios 9:3:6:80.5, similar to a previous synthesis reported by Krellner and Geibel [12] for CeRuPO single crystals. The $\mathrm{Fe}_{2} \mathrm{O}_{3}$ powder was dried for $\sim 10$ hours at $300^{\circ} \mathrm{C}$ before weighing. The ampoule was heated to $1135{ }^{\circ} \mathrm{C}$ at a rate of $35{ }^{\circ} \mathrm{C} / \mathrm{hr}$, kept at this temperature for 96 hours, and then rapidly cooled to $700{ }^{\circ} \mathrm{C}$. After removing the majority of the flux by spinning the ampoules in a centrifuge, LaFePO single crystal platelets of an isometric form with typical dimensions of $\sim 0.5 \times 0.5 \times 0.05 \mathrm{~mm}^{3}$ were collected and cleaned in hydrochloric acid to remove the flux from the surface of the crystals prior to measurements. After cleaning, the crystals were observed to have a distinct gold color. The platelets cleaved easily in the $a b$-plane and were notably malleable, in contrast to the cuprate superconductors. A typical platelet is shown in the inset of Fig. 1.

X-ray powder diffraction measurements were made using a Bruker D8 diffractometer with a non-monochromated $\mathrm{Cu} \mathrm{K} \alpha$ source to check the purity and crystal structure of the LaFePO single crystals. Due to their malleability, the crystals were difficult to grind into a 


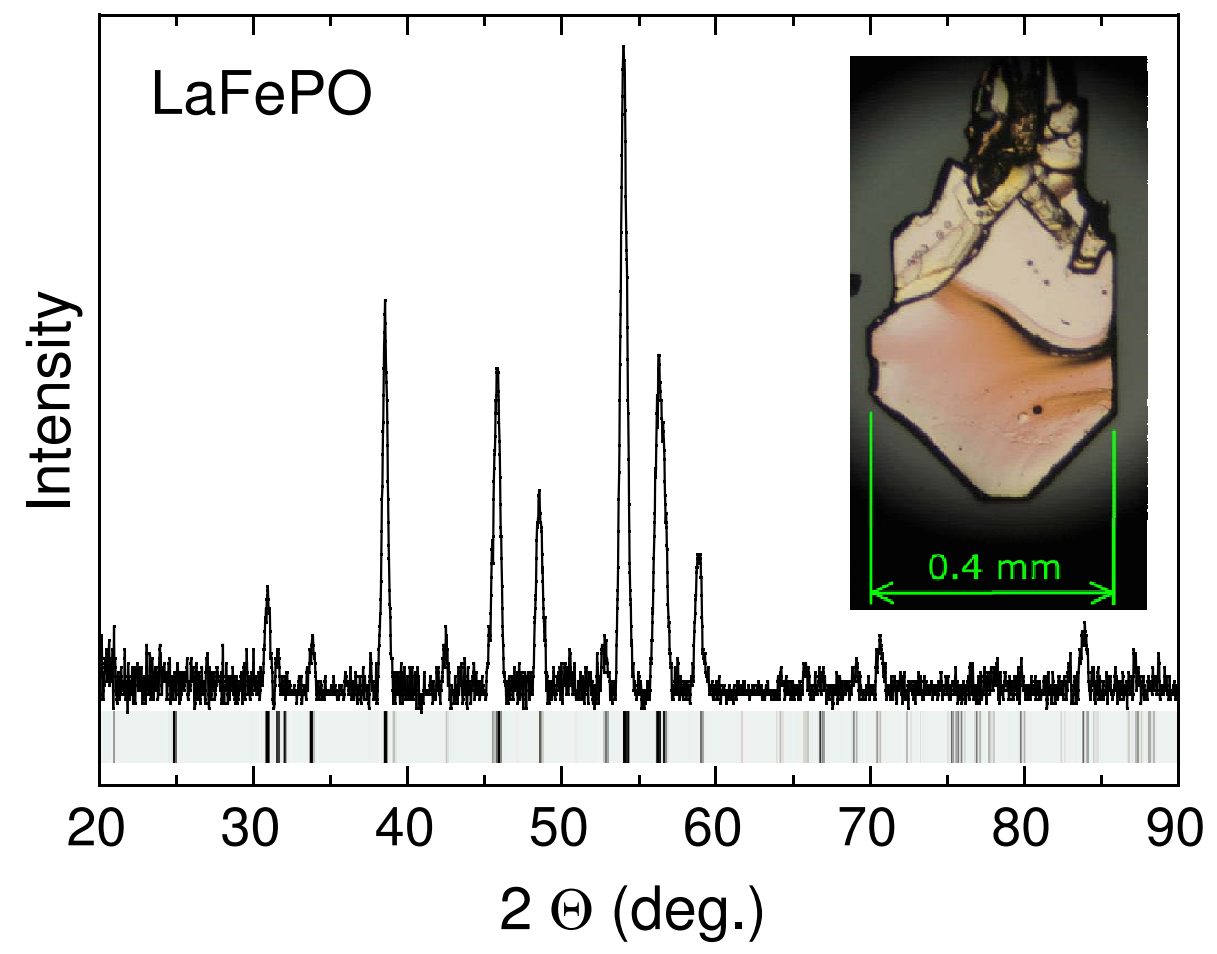

Figure 1. Powder $x$-ray diffraction pattern for LaFePO. The $y$-axis is linear. The vertical lines below the diffraction pattern indicate calculated Bragg peak positions. Inset: (color) Photograph shows a typical single crystal of LaFePO.

fine powder. Thus, the powder diffraction pattern was generated from a collection of several crystals which were cut into small pieces using a razor blade and then ground into a coarse powder using a mortar and pestle.

Electrical resistivity $\rho(T)$ measurements were performed in a four-wire configuration with the current in the $a b$-plane, at temperatures $T=2-300 \mathrm{~K}$ and magnetic fields $H=0-8$ $\mathrm{T}$ using a conventional ${ }^{4} \mathrm{He}$ cryostat and a Quantum Design Physical Properties Measurement System (PPMS). In order to explore the anisotropy of the superconducting properties, the resistivity was measured for $2-10 \mathrm{~K}$ with $H$ both parallel and perpendicular to the crystal $a b$-planes, $\rho\left(T, H_{\|}\right)$and $\rho\left(T, H_{\perp}\right)$, respectively. For these measurements, the geometry was arranged such that the current flowed in the $a b$-plane and was perpendicular to $H$, as illustrated in the upper panels of Fig. 3.

DC magnetization $M(T, H)$ measurements were made using a Quantum Design Magnetic Properties Measurement System (MPMS) in order to probe both the superconducting and normal state properties of the single crystal platelets. The specimens were mounted in cotton-packed gelatin capsules with the $a b$-plane perpendicular to the magnetic field. Multiple single crystal platelets were each individually measured for 2-10 K and $H=5$ Oe under both zero field cooled (ZFC) and field cooled (FC) conditions in order to characterize batch homogeneity via variation in $T_{c}$, which was found to be minimal. Magnetization versus temperature at $H=1.0 \mathrm{~T}$ and $7-300 \mathrm{~K}$ was measured for a collection of several hundred single crystal specimens ( $m=13.06 \mathrm{mg}$ ) in order to characterize the normal state. 


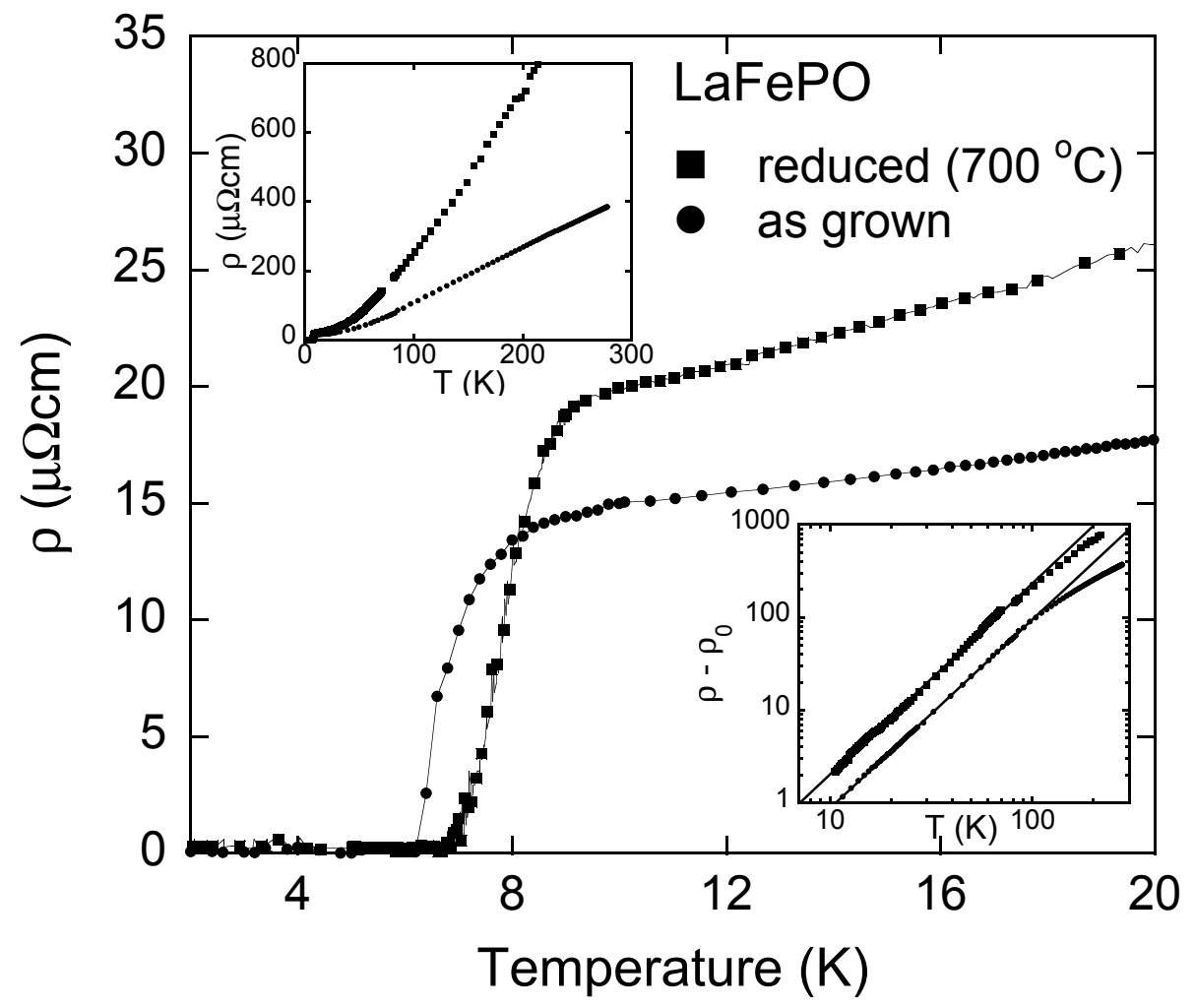

Figure 2. Electrical resistivity $\rho$ versus temperature $T$ measured in the $a b$-plane for an asgrown single crystal and a crystal that was reduced in flowing argon at $700{ }^{\circ} \mathrm{C}$ for $\sim 12$ hours. Left inset: Resistivity over the entire measured temperature range showing $T^{2}$ behavior for $\sim 10-100 \mathrm{~K}$ and linear $T$ behavior for $\sim 100-300 \mathrm{~K}$. Right inset: Log-log plot of $\rho-\rho_{0}$ versus $T$. The solid lines are fits to the data which demonstrate the $T^{2}$ behavior for $10-100 \mathrm{~K}$ for the as grown sample and $10-80 \mathrm{~K}$ for the reduced sample.

Specific heat $C(T)$ measurements were made for 2-300 K in a Quantum Design PPMS semiadiabatic calorimeter using a heat-pulse technique on the same collection of single crystals used for the normal state magnetization measurements. The specimens were attached to a sapphire platform with a small amount of Apiezon $\mathrm{N}$ grease.

A single high-pressure experiment was performed using a mechanically loaded commercial diamond anvil cell (DAC), manufactured by Kyowa Seisakusho Ltd. The diamond anvils were beveled from 500 to $250 \mu \mathrm{m}$ tips. One of the diamonds contains six deposited tungsten microprobes encapsulated in high-quality homepitaxial diamond. The fabrication of "designer" diamonds is described in Ref. [13]. The gasket was made from a $200 \mu \mathrm{m}$ thick MP35N foil pre-indented to $40-50 \mu \mathrm{m}$ with a $130 \mu \mathrm{m}$ diameter hole drilled through the center of the pre-indented region using an electrical discharge machine (EDM). Two $5 \mu \mathrm{m}$ diameter ruby spheres were loaded into the hole in the gasket and the remaining space in the hole was filled with $\sim 5$ small pieces of LaFePO crystal. Because the sample is in direct contact with the metallic gasket, the measured resistance results from a combination of the sample and gasket resistivity. Due to the geometry of the leads, the measured resistance generally does not drop completely to zero when the sample becomes superconducting. 
Temperature $(\mathrm{K})$
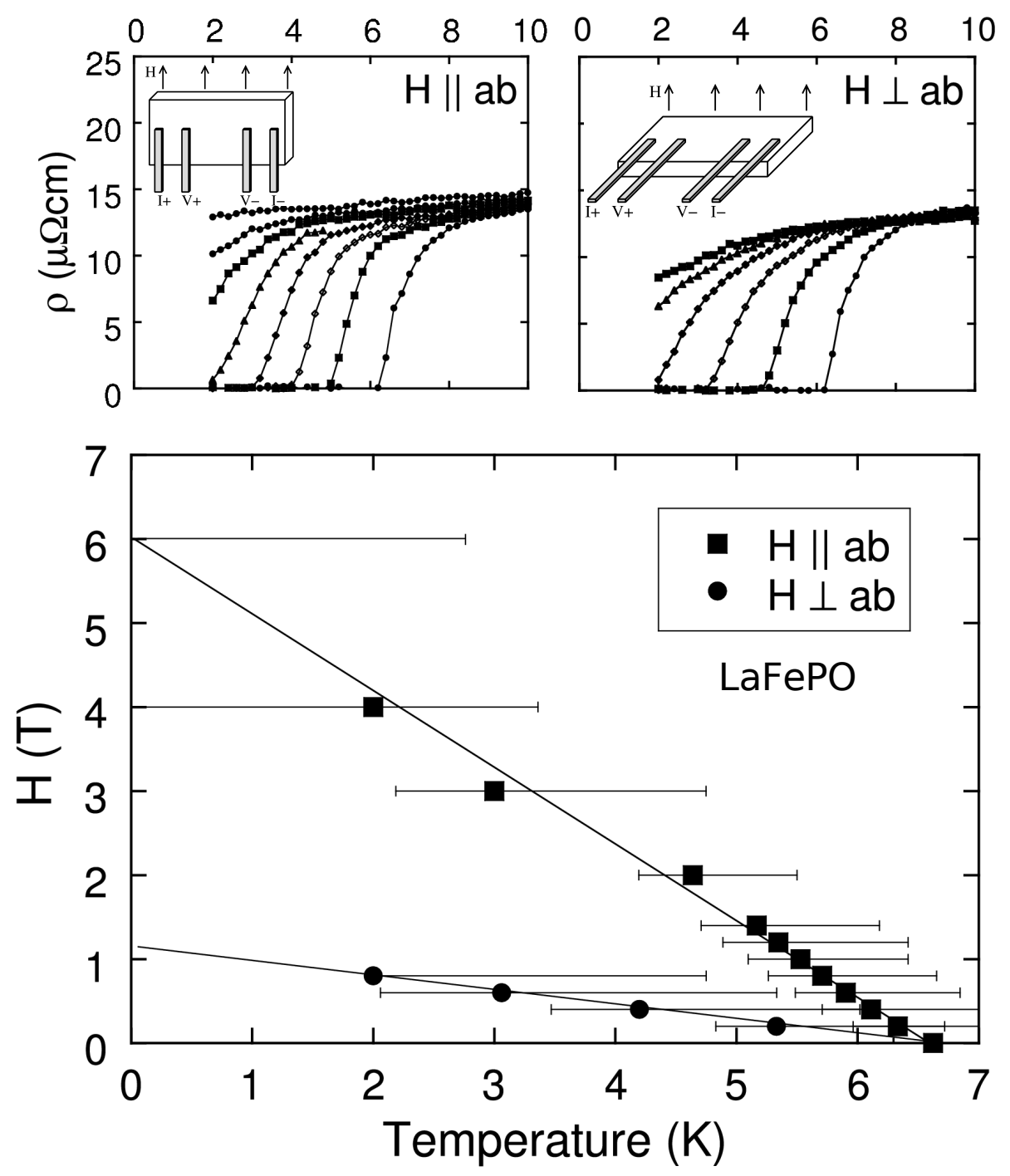

Figure 3. Top panels: Electrical resistivity $\rho$ versus temperature $T$ data for LaFePO single crystal platelets for temperatures $2-10 \mathrm{~K}$ and magnetic fields $\|$ to the $a b$-plane: $0 \mathrm{~T}, 1.0 \mathrm{~T}, 2.0$ $\mathrm{T}, 3.0 \mathrm{~T}, 4.0 \mathrm{~T}, 5.0 \mathrm{~T}, 6.0 \mathrm{~T}$, and $8.0 \mathrm{~T}$ (top left panel) and $\perp$ to the $a b$-plane: $0 \mathrm{~T}, 0.2 \mathrm{~T}, 0.4 \mathrm{~T}$, $0.6 \mathrm{~T}, 0.8 \mathrm{~T}$, and $1.0 \mathrm{~T}$ (top right panel). Bottom panel: The upper critical field line $H_{c 2}$ versus $T$ for $H \|$ and $\perp$ to the $a b$-plane.

However, such a configuration is sufficient for locating the $T_{c}$ of the sample. Pressure was adjusted and determined at room temperature, using the fluorescence spectrum of the ruby spheres and the calibration of Chijioke et al. [14]. A delrin spacer is used to minimize changes in pressure during cooling to low $T$. Additional details of the DAC technique are described in Ref. [15]. 


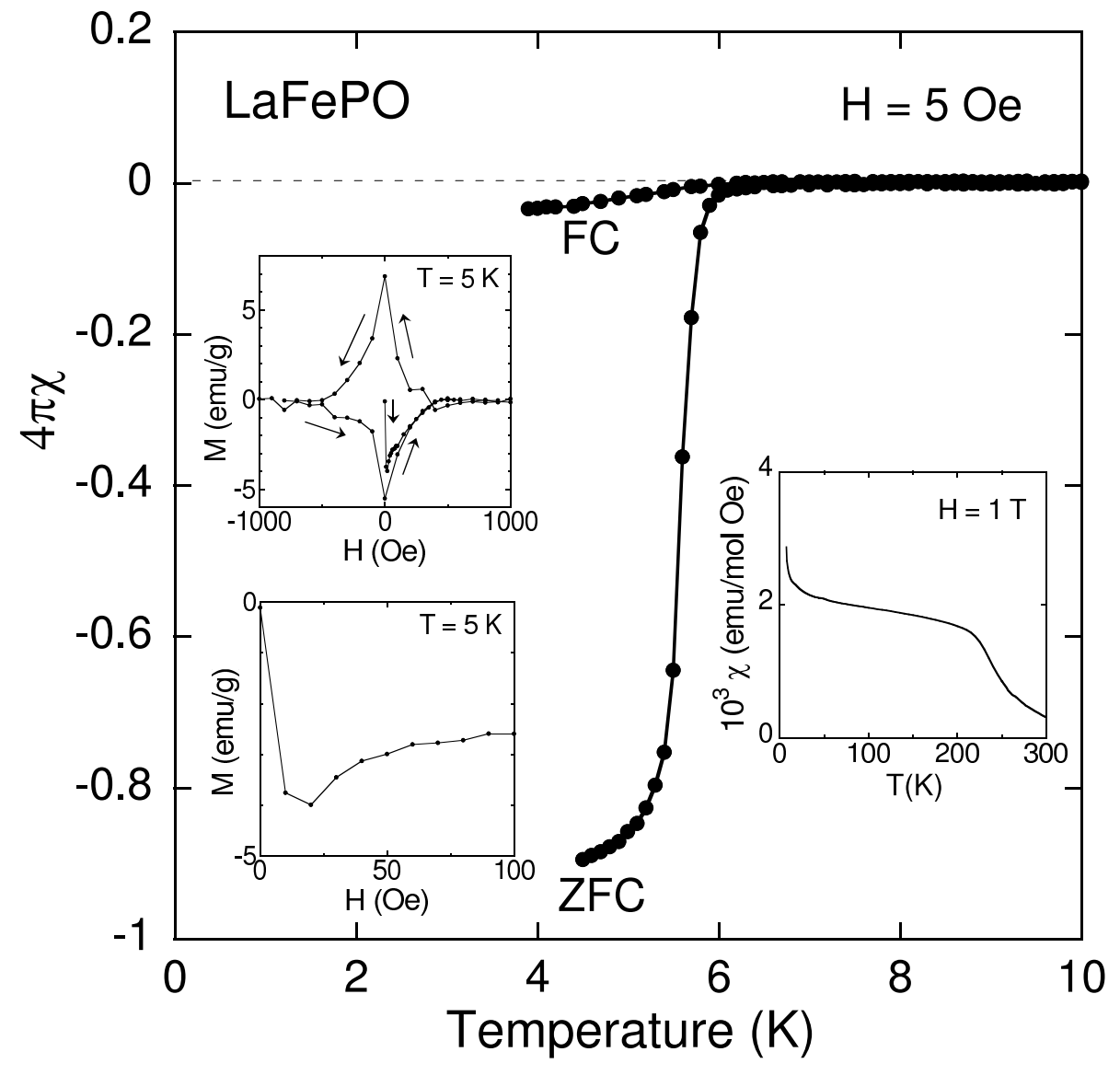

Figure 4. DC magnetic susceptibility $M / H$ versus temperature $T$ taken in $H=5$ Oe for a single crystal specimen under zero-field-cooled (ZFC) and field-cooled (FC) conditions. Left insets: $M$ versus $H$ exhibiting both flux expulsion (Meissner) and flux penetration (vortex) superconducting states as expected for a type-II superconductor. Right inset: $M / H$ versus temperature $T$ for 7-300 $\mathrm{K}$ and $H=1.0 \mathrm{~T}$.

\section{Results and Discussion}

Shown in Fig. 1 is the x-ray diffraction pattern for a collection of LaFePO single crystals. The diffraction pattern conforms to the characteristic tetragonal phase of $\mathrm{LaFePO}$ consisting of $\mathrm{P}-\mathrm{Fe}_{2}-\mathrm{P}$ layers of edge sharing $\mathrm{Fe}-\mathrm{P}$ octahedra separated by $\mathrm{La}-\mathrm{O}_{2}-\mathrm{La}$ sheets in which the Fe atoms form two-dimensional square nets. The diffraction pattern appears to be in good agreement with previous reports [7, 9, 11] and exhibits no impurity contributions down to the $10 \%$ level. Measurements on a mosaic of several single crystals reveal that the platelets grow with their large faces parallel to the $00 l$ crystal planes.

The electrical resistivity data, shown in Fig. 2, reveal metallic behavior where $\rho(T)$ decreases with decreasing $T$ until it drops abruptly to zero near the superconducting transition temperature $T_{c} \sim 6.6 \mathrm{~K}$. This transition temperature is defined as the temperature where $\rho(T)$ drops to $50 \%$ of its extrapolated normal state value. The transition width $\Delta T_{c}=1.3$ $\mathrm{K}$ is taken as the difference in the temperatures where $\rho(T)$ drops to $10 \%$ and $90 \%$ of 
the extrapolated normal state value. For $\sim 100-300 \mathrm{~K}, \rho(T)$ has an approximately linear $T$ dependence which evolves into a quadratic form for $\sim 10-100 \mathrm{~K}$, as shown in the left inset to Fig. 2. Fits over this temperature range show that the data are well described by the expression $\rho(T)=\rho_{0}+A T^{2}$ where $\rho_{0} \sim 14 \mu \Omega \mathrm{cm}$ and $A=9.46 \times 10^{-3} \mu \Omega \mathrm{cm} / \mathrm{K}^{2}$. The residual resistivity ratio $R R R=\rho(300 K) / \rho(0)=32$ reflects the high quality of the LaFePO single crystal. Also shown in Fig. 2 are results for a platelet which was reduced at $700{ }^{\circ} \mathrm{C}$ in flowing Ar for 24 hours. For this specimen, $R R R=58, T_{c}=7.8 \mathrm{~K}$, and the $\Delta T_{c}=1.6 \mathrm{~K}$, indicating that the superconducting state may be enhanced by reduction of oxygen concentration. Fits to the data for the reduced sample give $\rho_{0} \sim 16.7 \mu \Omega \mathrm{cm}$ and $A=2.44 \times 10^{-2} \mu \Omega \mathrm{cm} / \mathrm{K}^{2}$.

Shown in Fig. 3 are $\rho\left(T, H_{\|}\right)$and $\rho\left(T, H_{\perp}\right)$ data for $2-10 \mathrm{~K}$ and $0-8 \mathrm{~T}$ which reveal pronounced anisotropy in the upper critical field curve $H_{c 2}(T)$. Again, $T_{c}$ is defined as $50 \%$ of the extrapolated normal state value while the transition width is taken as the difference between the temperatures where $\rho(T)$ drops to $10 \%$ and $90 \%$ of the extrapolated normal state value. At zero $T$, the anisotropy is quantified by the ratio $H_{c 2}^{\|}(T) / H_{c 2}^{\perp}(T) \sim 5.2$. This result suggests that the layered structure strongly influences the in-plane and inter-plane transport behavior. The Clogston-Chandrasekhar [16, 17] Pauli-paramagnetic limiting field at zero temperature is given in units of tesla by $H_{p 0} \equiv 1.84 T_{c}$, which for $T_{c}=6.6 \mathrm{~K}$ gives $H_{p 0} \sim 12.1$ $\mathrm{T}$, a value well above the extrapolated zero temperature critical field line illustrated in Fig. 3 , Thus, the upper critical field is limited by orbital depairing for both $H \| a b$ and $H \perp a b$. The Ginzburg - Landau coherence lengths parallel and perpendicular to the $a b$-plane, $\xi_{\|}$and $\xi_{\perp}$, respectively, can be estimated from the slopes of $H_{c 2}^{\|}$and $H_{c 2}^{\perp}$ near $T_{c}$ [18], i. e.,

$$
\left(d H_{c 2}^{\perp} / d T\right)_{T_{c}}=-\Phi_{0} / 2 \pi T_{c} \xi_{\|}^{2}
$$

and

$$
\left(d H_{c 2}^{\|} / d T\right)_{T_{c}}=-\Phi_{0} / 2 \pi T_{c} \xi_{\perp} \xi_{\|},
$$

where $\Phi_{0}=h c / 2 e=2.07 \times 10^{-7} \mathrm{G} \cdot \mathrm{cm}^{2}$ is the flux quantum. From the values $\left(d H_{c 2}^{\perp} / d T\right)_{T_{c}}=$ $-1700 \mathrm{Oe} / \mathrm{K}$ and $\left(d H_{c 2}^{\|} / d T\right)_{T_{c}}=-8600 \mathrm{Oe} / \mathrm{K}$ we obtain $\xi_{\|}=170 \AA$ and $\xi_{\perp}=34 \AA$.

In an effort to determine whether the superconductivity is a bulk phenomenon, zerofield-cooled (ZFC) and field-cooled (FC) measurements of the magnetic susceptibility were made in a field of 5 Oe. A plot of the ZFC and FC magnetic susceptibility through the superconducting transition is shown in Fig. 4 where the onset temperature is near $6.0 \mathrm{~K}$. The ZFC and FC measurements yield maximum signals of $\sim 95 \%$ and $\sim 5 \%$ of complete flux expulsion, respectively. The values of $4 \pi \chi$ are accurate to roughly $\pm 15 \%$ due to uncertainties in mass, geometry and demagnetization factor of the small crystal. The small recovery of the diamagnetic signal on field-cooling indicates either that the material shows strong vortex pinning or that the superconductivity is not a bulk phenomenon and is possibly associated with regions in the crystal that are oxygen deficient. One such possibility is that the superconductivity resides in a region near the surface which has a composition different from that of the interior of the crystal. The left inset to Fig. 4 shows $M$ versus $H$ for $T=5$ $\mathrm{K}$, where the linear response of the Meissner state up to $\sim 20$ Oe is followed by a decrease in the magnitude of $M$ with increasing $H$, as is typical of flux penetration in the vortex state 
of a type-II superconductor. The $\chi(T)$ data in the normal state are shown in the right inset of Fig. 4. The susceptibility $\chi(T)$ increases strongly with decreasing $T$ down to $\sim 220 \mathrm{~K}$, where it partially saturates. This temperature dependence is similar to that seen for the compound $\mathrm{Fe}_{2} \mathrm{P}$ [20], which may be present as inclusions or surface impurities. We estimate that our observed magnetic susceptibility is consistent with $1-2 \%$ percent $\mathrm{Fe}_{2} \mathrm{P}$ impurity. It is notable that below $\sim 40 \mathrm{~K}, \chi(T)$ exhibits a weak upturn which persists down to $2 \mathrm{~K}$. This behavior is not typical for $\mathrm{Fe}_{2} \mathrm{P}$, and is either intrinsic to $\mathrm{LaFePO}$ or due to a small concentration of some other paramagnetic impurity.

Specific heat divided by temperature $C / T$ versus $T^{2}$ data for $2-20 \mathrm{~K}$ are shown in Fig. 5. The absence of a detectable jump in $C(T)$ near $T_{c} \sim 6.6 \mathrm{~K}$, strongly suggests that the LaFePO single crystals do not exhibit bulk superconductivity. The specific heat jump at $T_{c}$ for a weak-coupling conventional superconductor is given by $\Delta C=1.52 \gamma T_{c}$ [21]. For $T_{c}=6.6 \mathrm{~K}$ and $\gamma=12.7 \mathrm{~mJ} / \mathrm{mol} \cdot \mathrm{K}$, this relation yields $\Delta C=127 \mathrm{~mJ} / \mathrm{mol} \cdot \mathrm{K}$. Comparing this expected specific heat jump with the scatter in the $C(T)$ data (upper inset of Fig. 5) would indicate that at most $10-20 \%$ of the sample is superconducting. Our upper limit on the specific heat jump, $\Delta C \lesssim 13 \mathrm{~mJ} / \mathrm{mol} \cdot \mathrm{K}$, is consistent with a very recent measurement of Kohama et al. [22] on polycrystalline LaFePO, who find near $T_{c}$ a very small discontinuity of $\Delta C \sim 10$ $\mathrm{mJ} / \mathrm{mol} \cdot \mathrm{K}$. These results support the interpretation that the superconductivity is associated with defects such as oxygen vacancies that act to dope the compound or possibly a different surface composition in comparison with the bulk. On the other hand, LaFePO may exhibit some exotic type of superconductivity that is characterized by an unusually small energy gap.

The $C / T$ versus $T^{2}$ data shown in Fig. 5 can be fit, between $2 \mathrm{~K}$ and $8 \mathrm{~K}$, by the sum of electronic $\gamma T$ and lattice $\beta T^{3}$ terms, yielding an electronic specific heat coefficient $\gamma=12.7$ $\mathrm{mJ} / \mathrm{mol} \cdot \mathrm{K}^{2}$ and a Debye temperature $\Theta_{D}=268 \mathrm{~K}$. The value of $\gamma$ is close to $\gamma=12.5$ $\mathrm{mJ} / \mathrm{mol} \cdot \mathrm{K}^{2}$ obtained for a polycrystalline sample by McQueen et al. [11]. From the value $\gamma=12.7 \mathrm{~mJ} / \mathrm{mol} \cdot \mathrm{K}^{2}$ and the saturated magnetic susceptibility $\chi_{0} \sim 2 \times 10^{-3} \mathrm{emu} / \mathrm{mol} \cdot \mathrm{Oe}$, the Wilson - Sommerfeld ratio is calculated to be $\mathrm{R}_{W}=14.6$. By comparison to the values expected for noninteracting electrons and electrons in a bound state Kondo singlet, $\mathbf{R}_{W}=1$ and 2 , respectively, this value is unphysically large. This result indicates that the value of $\chi_{0}$ does not represent the Pauli paramagnetic susceptibility of the conduction electrons. For this reason, we consider the value of $\chi_{0}=3 \times 10^{-4} \mathrm{emu} / \mathrm{mol}$.Oe reported by McQueen et al. [11], which yields $\mathrm{R}_{W}=2.19$. This inconsistency is presumably due to the inclusion of $1-2 \%$ $\mathrm{Fe}_{2} \mathrm{P}$, which would enhance the assumed low $T$ susceptibility for conduction electrons. From the coefficient $A=9.46 \times 10^{-3} \mu \Omega \mathrm{cm} / \mathrm{K}^{2}$ obtained by fits to $\rho(T)$ and $\gamma=12.7 \mathrm{~mJ} / \mathrm{mol} \cdot \mathrm{K}^{2}$, the ratio $R \equiv A / \gamma^{2}=6 \times 10^{-5} \mu \Omega \mathrm{cm}(\mathrm{mol} \cdot \mathrm{K} / \mathrm{mJ})^{2}$ is calculated, in order of magnitude agreement with the Kadowaki - Woods value of $1 \times 10^{-5} \mu \Omega \mathrm{cm}(\mathrm{mol} \cdot \mathrm{K} / \mathrm{mJ})^{2}$ [23]. As shown in the lower inset to Fig. 5, $C(T) / T$ continues to increase rapidly up to $\sim 120 \mathrm{~K}$, where it goes through a peak and then decreases monotonically with increasing $T$.

Electrical resistivity $\rho(T)$ measurements were made under high pressures of 54, 106, 158, and 204 kbar, in that order, as shown in Fig. 6. The upper panels in Fig. 6 display the resistance versus pressure in the vicinity of the superconducting transition for two different configurations of the six tungsten leads. Each different configuration measures a somewhat 


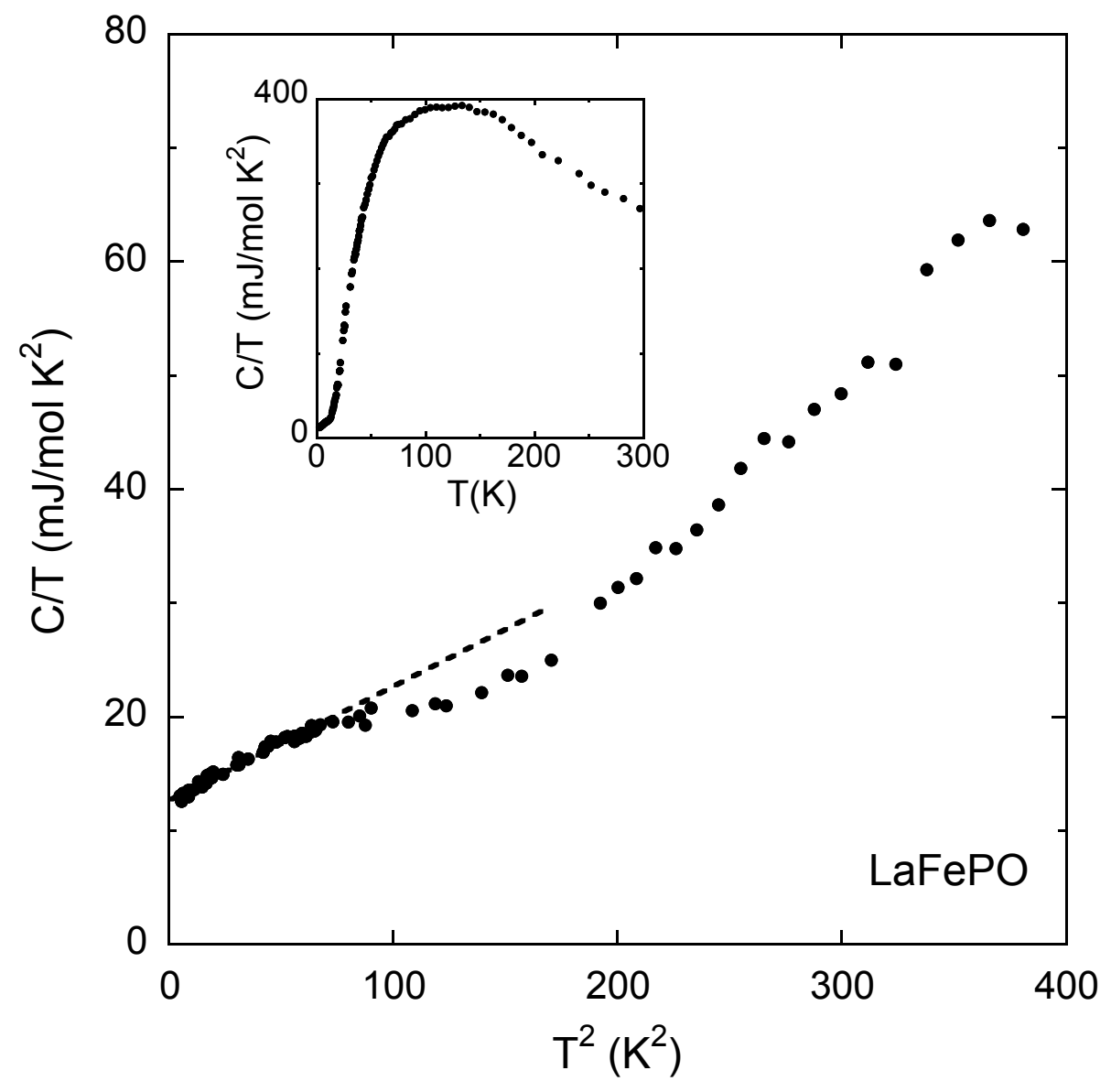

Figure 5. Specific heat $C$ divided by temperature $T, C / T$, versus $T^{2}$ for $2-20 \mathrm{~K}$. No discontinuity is observed near $T_{c}$, strongly suggesting that the superconductivity is not a bulk phenomenon. A linear fit (dashed line) to $C / T$ versus $T^{2}$ for $2-8 \mathrm{~K}$ yields an electronic specific heat coefficient $\gamma=12.7 \mathrm{~mJ} / \mathrm{mol} \cdot \mathrm{K}$ and a Debye temperature $\Theta_{D}=268 \mathrm{~K}$. Inset: $C(T) / T$ versus $T$ from $2-300 \mathrm{~K}$.

different section of the sample. The lower inset of Fig. 6 shows $\rho(T)$ over a broader temperature range for the measurement at $54 \mathrm{kbar}$. The $T_{c}$ values are determined graphically as illustrated by the dashed lines in the upper panels and averaged over the two configurations of leads. The values for the vertical bars represent the superconducting onset temperature and are determined by the temperature at which the resistivity passes through a maximum. The pressure is determined from an average of the pressure given by the two different pieces of ruby in the cell and the horizontal bars represent the pressure difference determined from each individual piece of ruby. The point at ambient pressure was taken from the resistivity curve shown in Fig. 2, Remarkably, a rather moderate pressure of 106 kbar is sufficient to nearly double the onset of superconductivity from $\sim 7 \mathrm{~K}$ to $\sim 14 \mathrm{~K}$, above which pressure acts to suppress $T_{c}$. 


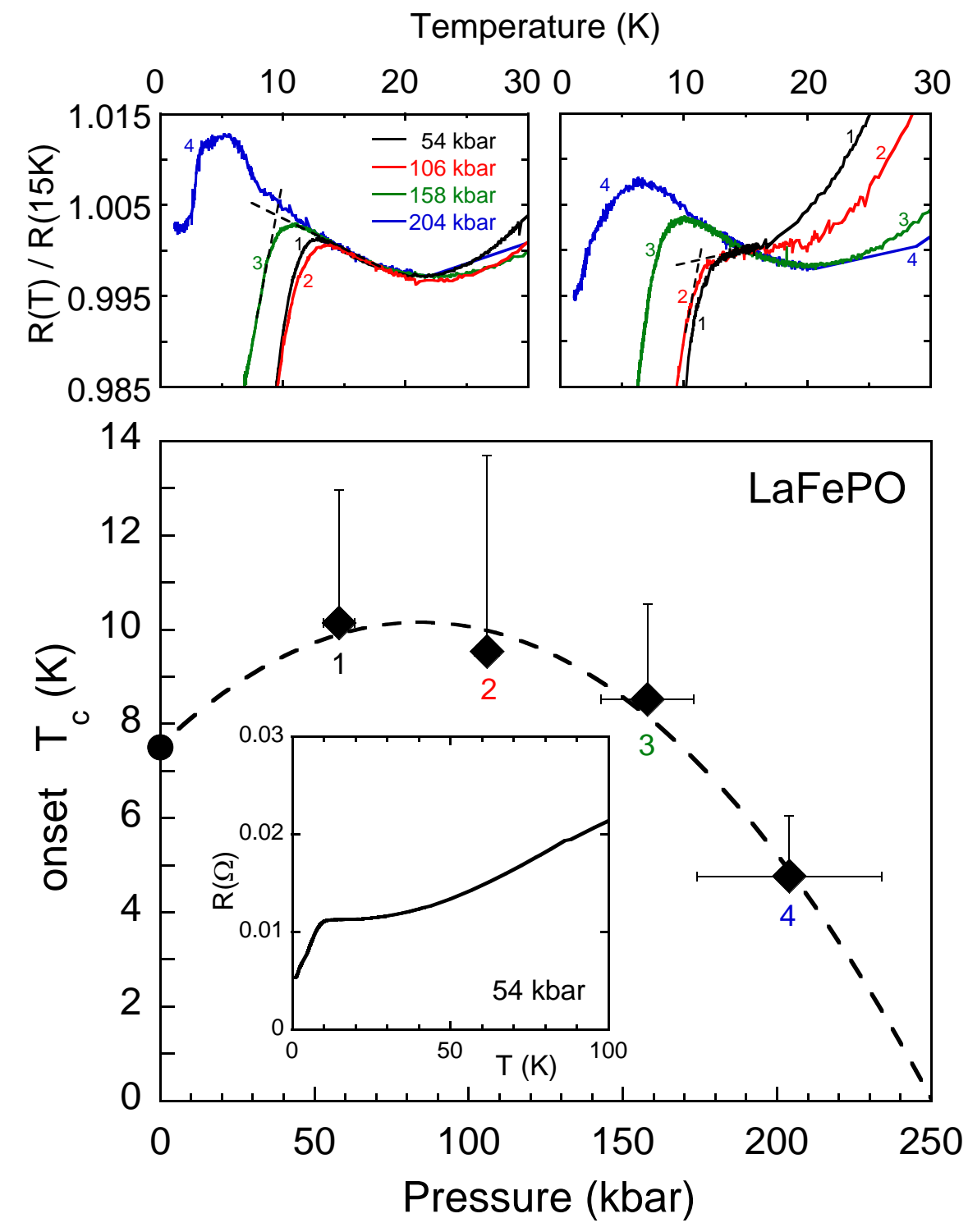

Figure 6. (color) Top panels: Resistance curves normalized to the values at $15 \mathrm{~K}$ for two different lead configurations where the onset $T_{c}$ is defined as the intersection of the dashed lines. Main panel: Pressure dependence of the onset $T_{c}$ for $\mathrm{P}=54,106,158$ and $204 \mathrm{kbar}$. The value of the $T_{c}$ onset at ambient pressure corresponds to the measurement of $R$ versus $T$ shown in Fig. 2 with $T_{c}$ determined using the same criterion as that used for the high pressure points as described above. The high-pressure points correspond to the DAC measurements. Horizontal bars represent the gradient of pressure along the sample, as described in the main text. The high temperature limit of the vertical bars represent an estimate of the first appearance of superconductivity, inferred from the first indication of the deviation of $\rho(T)$ from normal state behavior. The dashed line is a guide to the eye. 


\section{Concluding Remarks}

Single crystals of the compound LaFePO prepared by a flux growth technique at high temperatures exhibit a superconducting transition near $6.6 \mathrm{~K}$, as revealed by electrical resistivity and magnetization measurements. The transition temperature $T_{c}$ is suppressed anisotropically for magnetic fields perpendicular and parallel to the ab plane, suggesting that the crystalline anisotropy strongly influences the superconducting state. Measurements of electrical resistivity under pressure show that $T_{c}$ passes through a maximum of nearly $14 \mathrm{~K}$ at $\sim 110 \mathrm{kbar}$, indicating that significantly higher $T_{c}$ values may be possible in the phosphorus - based oxypnictides. In contrast, specific heat measurements show no indication of a discontinuity at $T_{c}$, suggesting that stoichiometric LaFePO does not exhibit bulk superconductivity. These results indicate that either the superconductivity is associated with oxygen vacancies that alter the carrier concentration, or that the superconductivity is characterized by an unusually small energy gap. Further experiments in which the compound is doped with charge carriers through chemical substitution will hopefully shed light on the superconductivity displayed by this new class of superconducting materials. Acknowledgements We thank S. T. Weir, D. D. Jackson, and Y. K. Vohra for providing designer diamonds. Thanks are also due J. R. Jeffries for setting up our diamond anvil cell facilities and O. Shpyrko for advice concerning x-ray diffraction. Research at University of California, San Diego, was supported by the U.S. Department of Energy grant number DEFG52-06NA26205, by the National Nuclear Security Administration under the Stewardship

Science Academic Alliance Program through the U.S. Department of Energy grant number DE-FG52-06NA26205, and the National Science Foundation grant number DMR0802478.

\section{References}

[1] Kamihara Y, Watanabe T, Hirano M, and Hosono H 2008 J. Am. Chem. Soc. 1303296

[2] Takahashi H, Igawa K, Arii K, Kamihara Y, Hirano M, and Hosono H 2008 Nature 453367

[3] Chen X H, Wu T, Liu R H, Chen H, and Fang D F 2008 Nature 453761

[4] Chen G F, Li Z, Wu D, Li G, Hu Z, Dong J, Zheng P, Luo J L, and Wang N L 2008 arXiv:condmat/0803.3790

[5] Ren Z, Lu W, Yang J, Yi W, Shen X, Li Z, Che G, Dong X, and Sun L 2008 arXiv:cond-mat/0804.2053

[6] Sefat A S, McGuire M A, Sales B C, Jin R, Howe J Y, and Mandrus D 2008 Phys. Rev. B 77174503

[7] Zimmer B I, Jeitschko W, Albering J H, Glaum R, and Reehuis M 1995 J. Alloys and Comp. 229238.

[8] Quebe P, Terbuchte L J, and Jeitschko W 2000 J. Alloys and Comp. 30270

[9] Kamihara Y, Hiramatsu H, Hirano M, Kawamura R, Yanagi H, Kamiya T, and Hosono H 2006 J. Am. Chem. Soc. 12810012.

[10] Tegel M, Schellenberg I, Pöttgen R, and Johrendt D 2008 arXiv:cond-mat/0805.1208

[11] McQueen T M, Regulacio M, Williams A J, Huang Q, Lynn J W, Hor Y S, West D V, Green M A, and Cava R J 2008 arXiv:cond-mat/0805.2149

[12] Krellner C and Geibel C 2008 J. of Crystal Growth 3101875

[13] Weir S T, Akella J, Aracne-Ruddle C, Vohra Y K, and Catledge S A 2000 Appl. Phys. Lett. 773400

[14] Chijioke A D, Nellis W J, Soldatov A, and Silvera I F 2005 J. Appl. Phys. 98114905

[15] Jackson D D, Jeffries J R, Qiu W, Griffiths J D, McCall S, Aracne C, Fluss M, Maple M B, Weir S T, and Vohra Y K 2006 Phys. Rev. B 74174401

[16] Clogston A M 1962 Phys. Rev. Lett. 9266. 
[17] Chandrasekhar B S 1962 Appl. Phys. Lett. 17

[18] see, for example, Salamon M B, Chapter 2, p. 43, in Physical Properties of Superconductors, Ginsberg D M, ed., (World Scientific, Singapore, 1989).

[19] Hake R R 1967 Appl. Phys. Lett. 10189

[20] Bellavance D, Mikkelsen J, and Wold A 1970 J. Solid State Chem. 2285

[21] Bardeen J, Cooper L N, and Schreiffer J R 1957 Phys. Rev. 1081175

[22] Kohama Y, Kamihara Y, Kawaji H, Atake T, Hirano M, and Hosono H, 2008 arXiv:cond-mat/0806.3139

[23] Kadowaki K and Woods S B, Solid State Commun. 58507 